\title{
The Effects of Conformational Variation on Structural Insights from Solution-Phase Surface- Enhanced Raman Spectroscopy
}

\author{
Mathieu L. Simeral ${ }^{1}$, Aobo Zhang ${ }^{1}$, Steven M. E. Demers ${ }^{1}$, Hannah J. Hughes ${ }^{1}$, Mohammad \\ Abdul-Moqueet ${ }^{2}$,Kathryn M. Mayer ${ }^{2}$, and Jason H. Hafner ${ }^{1,3}$
}

1. Department of Physics \& Astronomy, Rice University, Houston, TX

2. Department of Physics \& Astronomy, University of Texas at San Antonio, San Antonio, TX

3. Department of Chemistry, Rice University, Houston, TX

\section{SUPPORTING INFORMATION}

\section{SABERS Calculations}

SABERS is based on ratios of SERS and Raman peak intensities for two different vibrational modes. ${ }^{1}$ It begins with the unenhanced Raman scattering signal, $U_{n}$, for a specific vibrational mode $n$, adapted from the time-independent semiclassical theory of light scattering:

$$
U_{n}=k_{\lambda} N_{\text {Raman }} \frac{\left(\omega_{o}-\omega_{n}\right)^{4}}{\omega_{n}}\left\{\frac{45 a_{n}{ }^{2}+7 \gamma_{n}{ }^{2}}{45}\right\} \frac{1}{\left(1-e^{\left.h \omega_{n} / k_{B} T\right)}\right.} I_{o}
$$

where $k_{l}$ absorbs all fundamental constants and instrumental parameters such as the spectral efficiency of the grating and detector, $N_{\text {Raman }}$ is the number of molecules in the beam spot, $\omega_{\mathrm{o}}$ is the excitation frequency, $\omega_{n}$ is the Raman shifted frequency of the $n^{\text {th }}$ mode, $a_{n}$ and $\gamma_{n}$ are the average polarizability and average anisotropy Raman tensor invariants, respectively, $I_{\mathrm{o}}$ is the incident intensity, $k_{\mathrm{B}}$ is Boltzmann constant, and $T$ is the temperature. The SERS signal, $S_{n}$, for mode $n$ is calculated by applying field enhancement factors and using only the $a_{\mathrm{zz}}$ tensor component:

$$
S_{n}=k_{\lambda} N_{S E R S} \frac{\left(\omega_{o}-\omega_{n}\right)^{4}}{\omega_{n}} E_{o, n}^{2} E_{R, n}^{2} \alpha_{z z, n}^{2} \frac{1}{\left(1-e^{\left.h \omega_{n} / k_{B} T\right)}\right.} I_{o} .
$$


where $N_{\mathrm{SERS}}$ is the number of molecules at a nanoparticle end in the beam spot, and $E_{\mathrm{o}, n}$ and $E_{\mathrm{R}, n}$ are the field enhancements at the incident and Raman shifted wavelengths for the $n^{\text {th }}$ mode, respectively. ${ }^{2} \alpha_{z z}$ is the relevant tensor component since the near field is normal to the surface of the nanoparticle tip. This direction is defined as the $z$-axis and is, therefore, the direction of the incoming (near) field and the outgoing polarized scattering.

The field enhancements depend not only on the wavelength but also on the location of $n^{\text {th }}$ vibrational mode. We refer to this as the center of vibration $\left(C_{n}\right)$, calculated by taking the average of all $m$ atomic positions $\left(r_{n, m}\right)$ weighted by each atom's vibration amplitude $\left(A_{n, m}\right)$ :

$$
\vec{C}_{n}=\frac{\sum_{m} A_{n, m} \vec{r}_{n, m}}{\sum_{m} A_{n, m}}
$$

The electric field enhancements for each mode are calculated at these centers of vibration.

In order to remove unknowns, a first ratio is taken between $S_{n}$ and $U_{n}$ for a specific mode $n$ :

$$
r_{n}=\frac{N_{S E R S}}{N_{\text {Raman }}} E_{0, n}^{2} E_{R, n}^{2} \frac{\alpha_{z z, n}^{2}}{\left\{\frac{45 a_{n}^{2}+7 \gamma_{n}^{2}}{45}\right\}}
$$

This ratio removes instrumental constants, as well as the frequency and Boltzmann terms. The numbers of molecules in the beam spot $N_{\text {SERS }}$ and $N_{\text {Raman }}$ are also difficult to estimate, and usually represent a limiting factor in quantitative analysis of SERS. ${ }^{3,4} \mathrm{~A}$ second ratio $R_{2-1}$ between the $r_{n}$ values for two different modes removes them:

$$
R_{1-2}=\frac{S_{2} U_{1}}{U_{2} S_{1}}=\frac{E_{o, 2}^{2} E_{R, 2}^{2}}{E_{o, 1}^{2} E_{R, 1}^{2}} \frac{\alpha_{z z, 2}^{2}}{\alpha_{z z, 1}^{2}} \frac{\left(45 a_{1}^{2}+7 \gamma_{1}^{2}\right)}{\left(45 a_{2}^{2}+7 \gamma_{2}^{2}\right)}
$$

The second ratio, $R_{2-1}$, depends on the position and orientation of the molecule relative to the nanoparticle surface. $R_{2-1}$ can be measured experimentally from two peaks in the SERS and Raman spectra. It can also be calculated theoretically for different molecular positions and orientations, where the electric field enhancements are obtained from FEM calculations, and the Raman tensor 
components are calculated by TDDFT. To determine a structure, theoretical $R_{2-1}$ values of different molecular orientations and positions are compared to the experimental value.

\section{Spectral Peak Analysis}

Spectral peak intensities are taken to be the total number of CCD counts that make up a peak, but absorption and backgrounds must be carefully considered. Figure S1 displays a typical CCD image for a SERS spectrum of CTAB on gold bipyramids. The broad background covers 90 pixels vertically while the spectrum is concentrated in the center 10 pixels. The CCD counts are summed vertically over 90 pixels. The different sources of background are represented by the five spectra displayed in Figure $2 \mathrm{~b}$. The bias frame $(B F)$ was recorded at zero exposure time with no light. The $\mathrm{CCD}$ has a large bias count level, but it is flat and has low read noise. A dark frame $(D F)$ was recorded at the experimental exposure time with no light. Since the detector was cooled to -70C, the dark count was negligible so the dark frame is essentially identical to the bias frame. Two types of background were recorded in each experiment. $B G_{1}$ refers to the background spectrum recorded when the glass capillary is completely removed from the system. $B G_{2}$ refers to the spectrum with the capillary inserted but filled only with water. The sample spectrum (SP) includes the nanoparticle solution (SERS) or unenhanced surfactant or lipid solution (Raman) in the capillary.
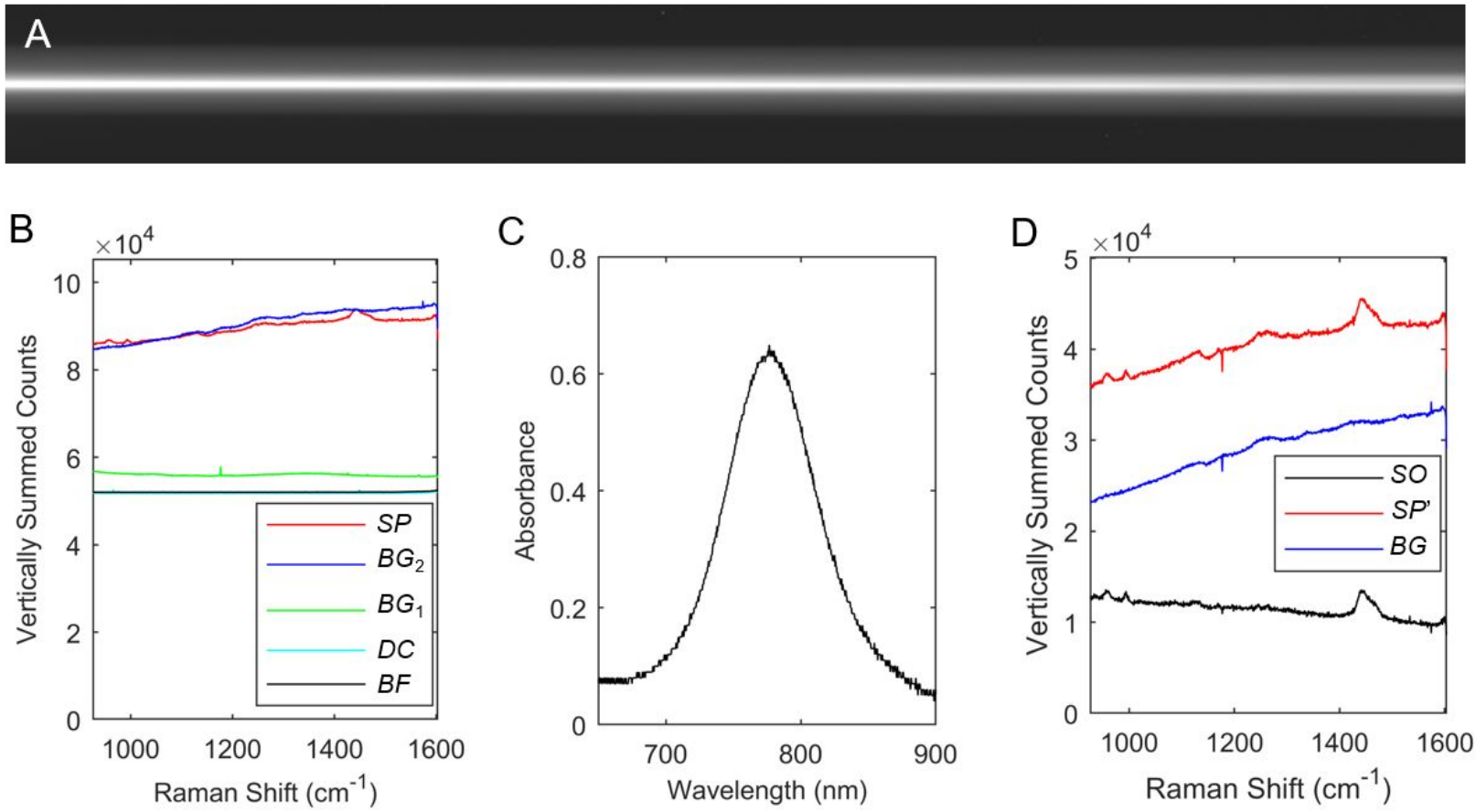
Figure S1. Processing the SERS and Raman spectra. (A) An example CCD image of a SERS spectrum in the $900-1600 \mathrm{~cm}^{-1}$ region. (B) The five spectra involved in each signal calculation: $S P=$ SERS or Raman spectra, $B G_{1}=$ background signal with no capillary, $B G_{2}=$ background signal with a water-filled capillary, $D C=$ dark count, and $B F=$ bias frame. (C) The gold bipyramid extinction spectrum that causes absorption of the signals. (D) The spectra and background after absorption correction and the resulting source $(S O)$ spectrum used for analysis.

As seen in Figure $\mathrm{S} 1 \mathrm{~b}$, the background signal $B G_{2}$ can be stronger than the nanoparticle spectrum. This is due to optical absorption of some background light by the nanoparticle sample, which does not occur in the background measurement. Accurate correction of the absorption effect requires separating the part of the background that is subject to absorption from the part that is not. $B G_{1}$ is not affected by absorption because it is due to light scattered inside the optical train, which does not pass through the sample or capillary at all. $B G_{2}$, which is due to reflections and emission from the glass capillary walls, is partially subject to absorption since about half of the background light is from the front glass wall (does not pass through absorbing sample) and about half is from the back glass wall (passes through the absorbing sample).

In order to correct for absorption, a spectral extinction measurement was recorded with the sample in a $1 \mathrm{~cm}$ path cuvette (displayed in Figure S1c), which yields the absorbance at the excitation wavelength $\left(A_{785}\right)$ as well as all Raman shifted wavelengths $(A) . B G_{1}$ was subtracted from $B G_{2}$, and that difference was modified by an expression which represents the absorption by the sample:

$$
\begin{gathered}
B G_{2}{ }^{\prime}=B G_{2}-B G_{1} \\
B G=\frac{1}{2} B G_{2}^{\prime}+\frac{1}{2} B G_{2}^{\prime} / 10^{\left(0.1 A_{785}+0.1 A\right)}
\end{gathered}
$$

The absorption correction lowers half of the background signal to the level that would occur if the absorbing nanoparticle sample were in the path. There are two absorption terms in Equation 2: one for the extinction of the excitation beam at $785 \mathrm{~nm}\left(A_{785}\right)$ and one for the absorption at the wavelength of each pixel $(A)$. The factor of 0.1 represents the $1 \mathrm{~mm}$ path length of the capillary relative to the $1 \mathrm{~cm}$ path length of the absorption measurement. The corrected background is subtracted from the spectrum, $S P$, to find the signal from the nanoparticle scattering $S P^{\prime}$. However, this must also be corrected (increased) to account for absorption: 


$$
\begin{gathered}
S P^{\prime}=S P-B G \\
S O=S P^{\prime} \times 10^{\left(0.05 A_{785}+0.05 A\right)}
\end{gathered}
$$

Here the two absorption terms again represent the excitation wavelength $\left(A_{785}\right)$, and the Raman shifted wavelength $(A)$. The factor 0.05 presumes that the absorption occurs through a path that is halfway through the capillary. $S O$ is the fully corrected source spectrum, displayed in Figure S1d, that was used to determine intensities of individual peaks.

The corrections described above resulted in a relatively flat background, but it was still necessary to remove the slope due to the SERS continuum, due to nearby peaks, or due to imperfect background removal..$^{5}$ To analyze an individual peak, a first or second-order polynomial fit was applied to a $100 \mathrm{~cm}-1$ region with the peak itself (about $30 \mathrm{~cm}-1$ ) blocked from the fit. The process is illustrated in Figure S2a where the red points are the background data used in the fit, and the black points are the blocked peak. The flattened peak was then fit to a Lorentzian curve (see Figure $\mathrm{S} 2 \mathrm{~b})$. The peak's intensity was taken to be the sum of CCD counts in the blocked region. For uncertainty, Poisson statistics were assumed so that the uncertainty is the square root of the counts at each pixel. Uncertainty in the peak intensity was taken as the square root of the number of counts at each pixel, and then combined. 

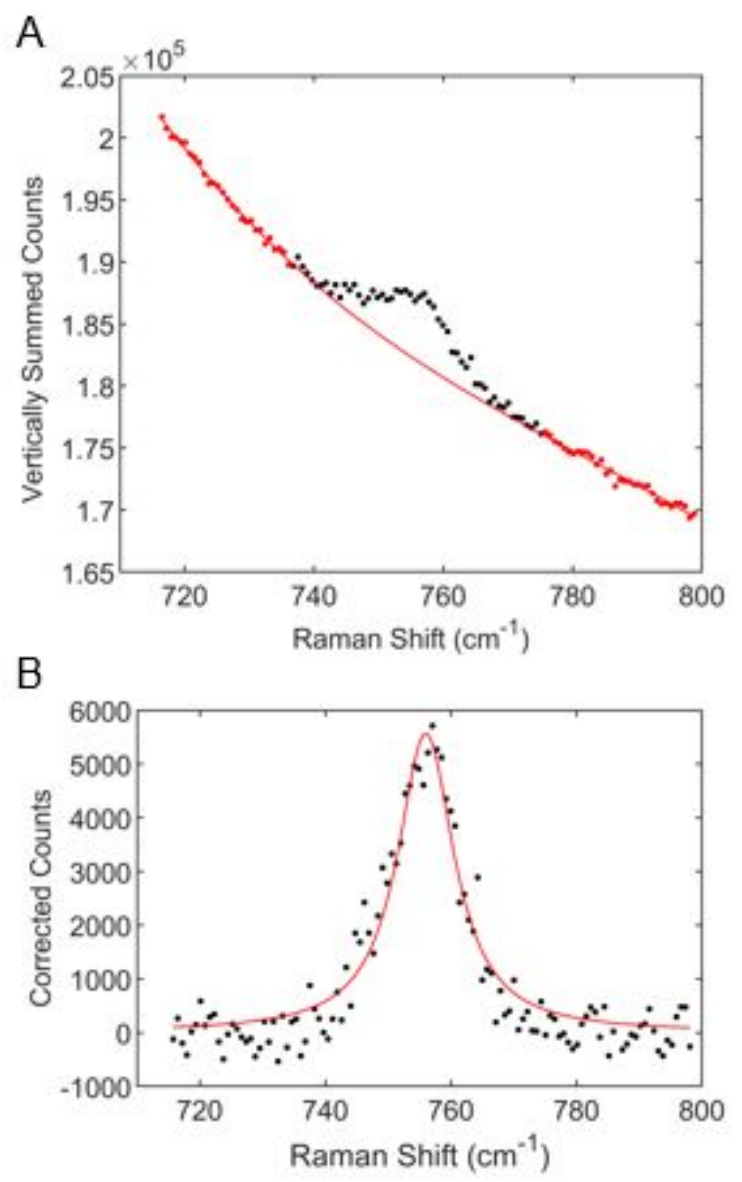

Figure S2. Peak fitting procedure. (A) The data used (red points) and the data blocked (black points) for background polynomial fitting are indicated, as well as the polynomial fit (red line). (B) The SERS peak after background subtraction, with Lorentzian fit.
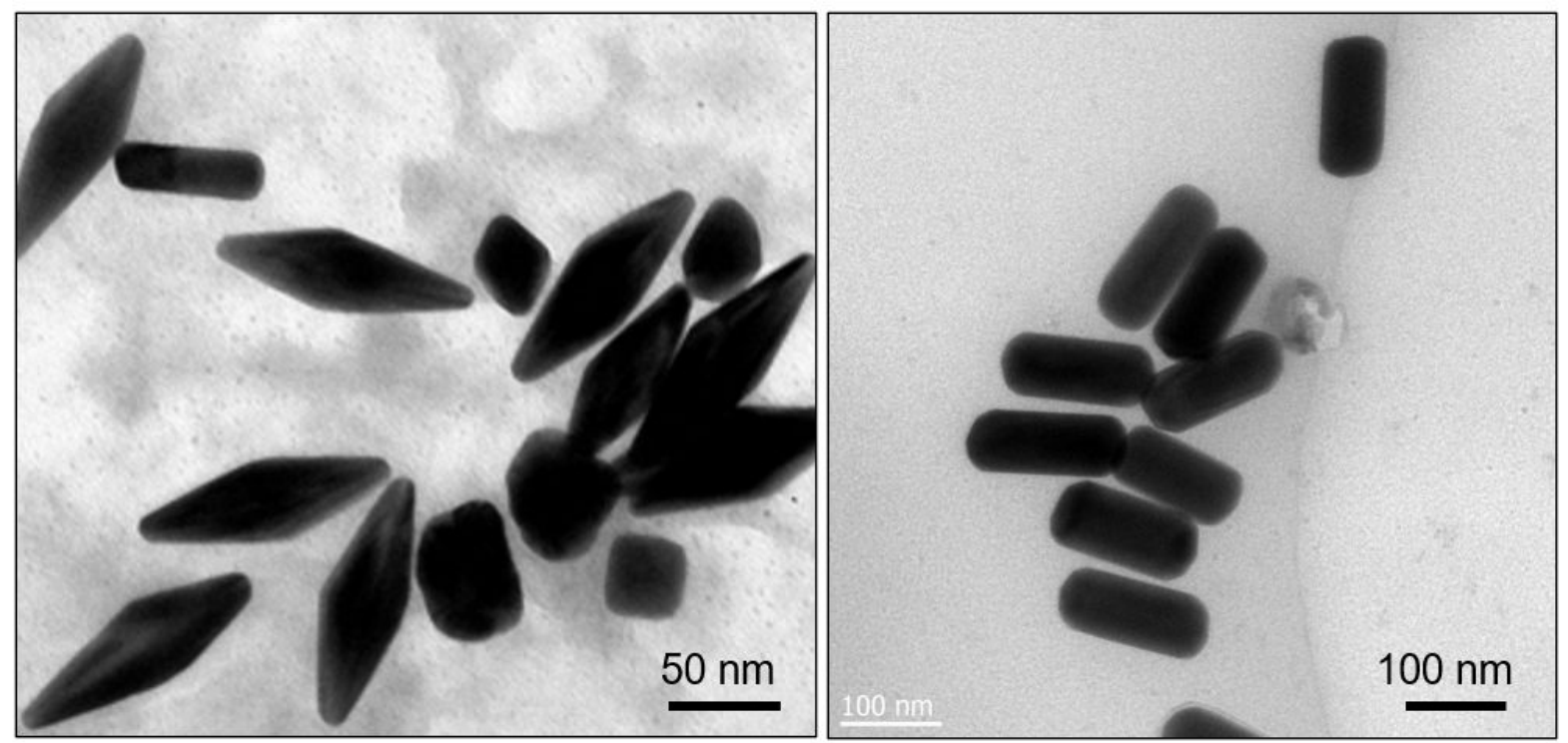
Figure S3. Representative TEM image of the gold bipyarmids (left) and gold nanorods (right) used for these experiments.

\section{REFERENCES}

(1) Matthews, J. R.; Shirazinejad, C. R.; Isakson, G. A.; Demers, S. M. E.; Hafner, J. H. Structural Analysis by Enhanced Raman Scattering. Nano Lett. 2017, 17 (4), 2172-2177. https://doi.org/10.1021/acs.nanolett.6b04509.

(2) Long, D. A. The Raman Effect: A Unified Treatment of the Theory of Raman Scattering by Molecules, 1st Edition.; Wiley: Chichester; New York, 2001.

(3) Lee, S.; Anderson, L. J. E.; Payne, C. M.; Hafner, J. H. Structural Transition in the Surfactant Layer That Surrounds Gold Nanorods as Observed by Analytical SurfaceEnhanced Raman Spectroscopy. Langmuir 2011, 27 (24), 14748-14756. https://doi.org/10.1021/la202918n.

(4) Le Ru, E. C.; Blackie, E.; Meyer, M.; Etchegoin, P. G. Surface Enhanced Raman Scattering Enhancement Factors: A Comprehensive Study. J. Phys. Chem. C 2007, 111 (37), 13794-13803. https://doi.org/10.1021/jp0687908.

(5) Hugall, J. T.; Baumberg, J. J. Demonstrating Photoluminescence from Au Is Electronic Inelastic Light Scattering of a Plasmonic Metal: The Origin of SERS Backgrounds. Nano Lett. 2015, 15 (4), 2600-2604. https://doi.org/10.1021/acs.nanolett.5b00146. 